\title{
Concentrations of Nicotinamide in Plasma by RP-HPLC With Fluorescence Detection
}

\author{
Zhipeng Pan ${ }^{1}$, Da Li ${ }^{2}$, Xiaokun Yao ${ }^{1}$, Shisheng Zhou ${ }^{1}$ and Yongzhi Lun ${ }^{1, a}$ \\ ${ }^{1}$ Liaoning Provincial University Key Laboratory of Biophysics, College of Medicine, Dalian University, Dalian, Liaoning 116622, P.R. \\ China \\ ${ }^{2}$ Department of Obstetrics and Gynecology, Shengjing Hospital of China Medical University, Shenyang, Liaoning 110004, P.R. China
}

\begin{abstract}
The purpose of this study is to establish a new method for detecting nicotinamide concentration in plasma. In the experiment, the high performance liquid chromatography (HPLC) method was used, with a fluorescence detector. The nicotinamide in the plasma was first converted to N1- methylnicotinamide, then reacted with acetophenone under certain conditions to produce fluorescent derivatives for testing. The method is a kind of highly sensitive detection, of which the lower limit is $10 \mathrm{ng} / \mathrm{mL}$, the recovery rate is between $92.75 \%$ and $105.13 \%$, and the relative standard deviation (RSD) is between $3.76 \%$ and $4.43 \%$. The results showed that this measurement method is accurate, sensitive and rapid. It meets the requirements of the experiment, and applies to the detection of nicotinamide concentration in plasma.
\end{abstract}

Nicotinamide (NM), namely Nike amide, vitamin B3 and vitamin $\mathrm{PP}$, is a water-soluble vitamin(Figure 1$)^{[1]}$. Nicotinamide is an alkylated derivative of nicotinic acid, which is also the precursor of coenzyme I (NAD) and coenzyme II (NADP) in the body, thus the pharmacokinetic observation of nicotinamide is an important tool for monitoring metabolism levels in the body ${ }^{[2]}$. Our research shows that long-term nicotinamide overload can cause insulin resistance and oxidative stress, and eventually lead to diabetes ${ }^{[3,4]}$. Currently, the detection of nicotinamide in plasma samples is a complex method, which requires high instrument conditions and liquid chromatography - mass spectrometry ${ }^{[5-7]}$. This method is also highly timeconsuming, mainly due to the very low levels of nicotinamide in the plasma. In this study, concentrations of nicotinamide were measured using the ordinary highperformance liquid chromatography method with cleverly designed, simple processing and saving supplies, and satisfactory results were achieved ${ }^{[8-14]}$.

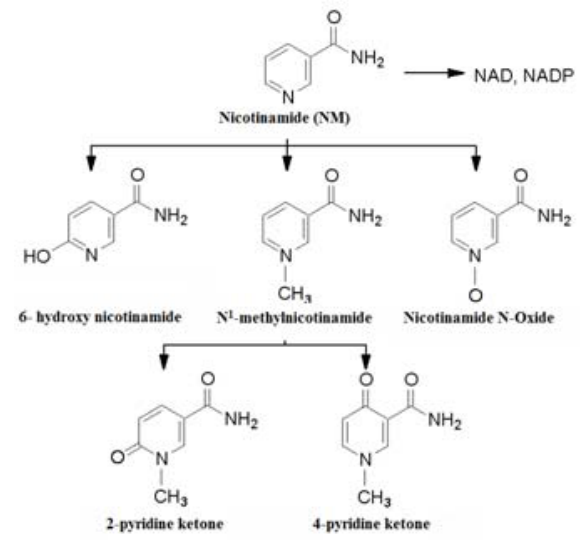

Figure 1. Vitamin PP metabolism in human

\section{Methods}

1.1 Instruments: High-performance liquid chromatography (Shimadzu LC-9A pump, Rheodyne $7725 \mathrm{i}$ injector, $20 \mu \mathrm{L}$ sample loop, Shimadzu Co.), Waters470 fluorescence detector (Waters, USA), N2000 chromatography workstation (Zhejiang University Zhida Information Engineering Co., Ltd.).

1.1.1 Reagents: Ethyl acetate (AR), acetophenone (AR), formic acid (AR), $\mathrm{NaCl}$ (Beijing Jingke Hongda), $0.5 \mathrm{M}$ $\mathrm{HCl}$; 0.5M NaH2PO4, 0.4M KOH, 20\% trichloro acetic acid (TCA), Nicotinamide (NM), and N1methylnicotinamide (NMN) (Japan Takeda Co.). N1ethylnicotinamide (NEN) was synthesized according to the methods described by Hirayama et al. ${ }^{[15,16]}$, with a purity of $99.99 \%$, and this was used as an internal standard.

1.2 Chromatographic conditions: The column in the experiments: ODS-C18 column (Thermo, Bellefonte, PA), $250 \mathrm{~mm} * 4.6 \mathrm{~mm}$ (id), particle size $5 \mu \mathrm{m}$; mobile phase: $10 \mathrm{mM}$ heptane sulfonate, $50 \mathrm{mM}$ triethylamine solution $(\mathrm{pH} 3.2)$ : acetonitrile $(78: 22 \mathrm{v} / \mathrm{v})$; excitation wavelength $366 \mathrm{~nm}$, emission wavelength $418 \mathrm{~nm}$; flow rate $1.0 \mathrm{~mL} / \mathrm{min}$; injection volume $20 \mu \mathrm{L}$.

1.3 Sample preparation: $200 \mu \mathrm{L}$ blood was input into the EP tube with $100 \mu \mathrm{L} 20 \%$ TCA and then mixed by vortex, and $12000 \mathrm{rpm}$ of centrifugation for $10 \mathrm{~min}$; next, $200 \mu \mathrm{L}$ supernatant was removed and placed in a new EP tube. $25 \mu \mathrm{L} 0.5 \mathrm{M} \mathrm{HCl}$ was added with $100 \mathrm{mg} \mathrm{NaCl}$ into the supernatant, then shocked by vortex until the water phase was fully saturated by the $\mathrm{NaCl}$, which was extracted three times by $1 \mathrm{~mL}$ of water-saturated ethyl acetate extract, then the upper phase of ethyl acetate was

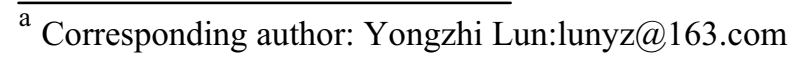


discarded and the residual ethyl acetate was blow-dried with $\mathrm{N}_{2} .25 \mu \mathrm{L} 0.5 \mathrm{M} \mathrm{NaH} \mathrm{PO}_{4}$ and $25 \mu \mathrm{L} 0.4 \mathrm{M} \mathrm{KOH}$ were continuously added, and these were shocked and mixed well, followed by the addition of $1 \mathrm{~mL}$ anhydrous ethyl acetate, which was shocked and mixed. Centrifugation was performed at $3000 \mathrm{rpm}$ for $3 \mathrm{~min}$, then the $800 \mu \mathrm{L}$ upper phase was taken in another EP tube, followed by $\mathrm{N}_{2}$ drying ${ }^{[17]}$. $250 \mu \mathrm{L}$ iodomethane was added and the two were mixed together, then saved in dark for $24 \mathrm{~h}$ before being dried with $\mathrm{N}_{2}$. Then $200 \mu \mathrm{L}$ double distilled water was added, and extracted three times with ethyl acetate saturated with water after the shocking; the residue in the ethyl acetate was dried by $\mathrm{N}_{2}$, at which point all of the nicotinamide in the plasma was converted into the N1-methylnicotinamide. $200 \mu \mathrm{L}$ internal standard was added $(50 \mathrm{ng} / \mathrm{mL})$, then $200 \mu \mathrm{L}$ of $100 \mathrm{mM}$ acetophenone was added ${ }^{[18]}$, and placed in the ice bath for $10 \mathrm{~min}$ after shocking. $400 \mu \mathrm{L}$ of $6 \mathrm{M} \mathrm{NaOH}$ was added and mixed well, then placed in the ice bath for $60 \mathrm{~min}$. Then $200 \mu \mathrm{L}$ of formic acid was added, and placed in the ice bath for $60 \mathrm{~min}$. Finally, the solution was placed in the boiling water bath for $3 \mathrm{~min}$ to generate the fluorescent derivatives, then mixed well after cooling for machine testing.

1.3.1 Determination: using the internal standard method and analyzed by HPLC with fluorescence detection, and the N2000 data acquisition system collected the data and calculated the concentration of nicotinamide in the plasma. The nicotinamide concentration was calculated according to the following formula[Equation (1)] based on N1-methylnicotinamide determination:

$$
\mathrm{m}=9.375 \mathrm{~m}_{0} \times \mathrm{M} / \mathrm{M}_{0}
$$

Where $\mathrm{m}$ is the concentration of nicotinamide in the plasma, unit: $\mathrm{ng} / \mathrm{ml} ; \mathrm{m}_{0}$ is the mass of the $\mathrm{N1-}$ methylnicotinamide, unit: nanograms (ng); $\mathrm{M}_{0}$ is the molecular weight of the N1-methylnicotinamide, molecular weight: 172.61; and $\mathrm{M}$ is the molecular weight of the nicotinamide, molecular weight: 122.12 .

\section{Results}

2.1 Chromatogram of nicotinamide in plasma: Figure. 2 is a blank of the chromatogram of the nicotinamide in the plasma, and Figure. 3 is a marked chromatogram of nicotinamide in the plasma. Peak 1 of the chromatogram is nicotinamide, and peak 2 is the internal standard in the figure.

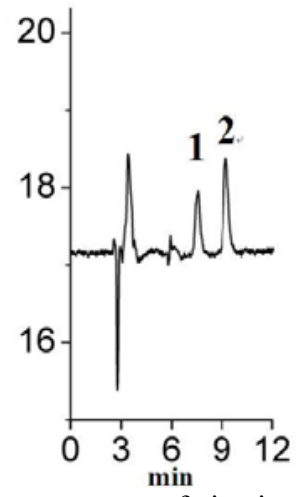

Figure.2 Chromatogram of nicotinamide in plasma

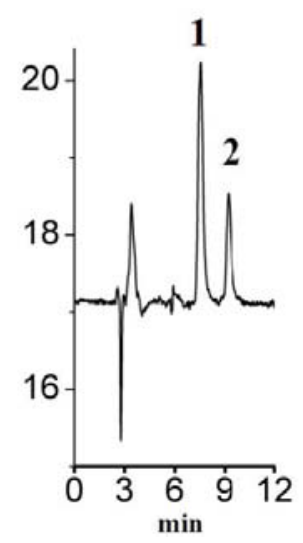

Figure.3 Marked chromatogram of nicotinamide in plasma 2.2 The minimum detection limit: the minimum detection limit for the RP-HPLC fluorescence detection method in the experimental conditions and chromatographic conditions, according to the peak response value of 3 times of the noise, the sample volume is calculated as the minimum detectable amount $[19,20]$. The detection lower limit of this method is 10 $\mathrm{ng} / \mathrm{mL}$.

2.3 The recovery rate and precision of the experiment: Three plasma samples were treated using the same method, once per day for 6 days. The results are as shown in Table 1.

Table 1. Precision experiments among batches $(n=6)$

\begin{tabular}{|c|c|c|c|c|c|}
\hline $\begin{array}{r}\text { Sample* } \\
\mathrm{X}+\mathrm{SD}\end{array}$ & $\begin{array}{l}\text { Number Measured } \\
\operatorname{RSD}(\%)\end{array}$ & value (ng & $\mathrm{mL})$ & & \\
\hline $\begin{array}{l}\text { Sample1 } \\
159.16 \pm 18.86\end{array}$ & $\begin{array}{l}158.05, \quad 168.67, \\
4.43\end{array}$ & 175.58 & 148.47, & 154.76, & 149.43 \\
\hline $\begin{array}{l}\text { Sample2 } \\
158.84 \pm 10.72\end{array}$ & $\begin{array}{l}161.40, \quad 140.98, \\
4.38\end{array}$ & 154.97, & 156.69, & 168.46, & 170.53 \\
\hline $\begin{array}{l}\text { Sample3 } \\
144.75+9.21\end{array}$ & $\begin{array}{ll}141.65, & 138.79, \\
3.76 & \end{array}$ & 159.85, & 144.19, & 133.77, & 150.23 \\
\hline
\end{tabular}

Precision and recovery rate experiment among batches: taking 3 parts of normal human plasma, which is divided into three parts after mixing, the standard of nicotinamide was added by the concentration gradient, according to the same procedure used to deal with the samples, which were then detected. Under the same conditions, each sample was continuously injected six times, and according to the corresponding peak area to calculate the RSD, the experimental results are as shown in Table 2.

Table 2. Recoveries and precision $(\mathrm{n}=6)$

\begin{tabular}{|c|c|c|c|}
\hline $\begin{array}{c}\text { Sample ba } \\
\text { recovery value }\end{array}$ & $\begin{array}{l}\text { ackground value } \\
\text { recovery rate }\end{array}$ & $\begin{array}{l}\text { marked value } \\
\text { RSD }\end{array}$ & measured value \\
\hline Sample4 & 153.67 & 100.00 & 246.42 \\
\hline 92.75 & 92.75 & 4.72 & \\
\hline Sample4 & 153.67 & 200.00 & 363.93 \\
\hline 210.26 & 105.13 & 3.94 & \\
\hline Sample4 & 153.67 & 500.00 & 635.57 \\
\hline 481.90 & 96.38 & 3.27 & \\
\hline
\end{tabular}

\section{Discussions}

3.1 The high performance liquid chromatography with fluorescence detector was used in this paper to measure the concentration of nicotinamide in the plasma. Its 
operation is simple and reproducible, with a high recovery rate, and the experimental results were satisfactory compared with those of other liquid chromatography-mass spectrometry methods, showing that it provides an experimental method for the study of pharmacokinetics and dynamics of nicotinamide.

3.2 In the sample processing, it is necessary to be careful when shaking with ethyl, otherwise the ethyl may overflow, possibly causing the loss of nicotinamide, thus causing the results to decrease in accuracy. When N1methylnicotinamide was converted into nicotinamide, the saturated ethyl acetate in the acidic conditions can be extracted to the original N1-methylnicotinamide of plasma, but this cannot be done under neutral conditions, thus it is important to pay particular attention to the $\mathrm{pH}$ value. The final amount of N1-methylnicotinamide was solely converted from the nicotinamide, thus the final amount of N1-methylnicotinamide is equal to the nicotinamide.

3.3 Due to the fact that the concentration levels of nicotinamide in the body differ among people, the nicotinamide content of samples 1,2 and 3 in this experiment are different. Nicotinamide is easily oxidized in air to form oxidized nicotinamide, thus it is necessary to pay particular attention to sample storage; the samples should be stored in a $-20^{\circ} \mathrm{C}$ refrigerator, and processed and detected promptly after treatment.

\section{Acknowledgments}

We would like to express our thanks to the following projects for funding this study: the National Natural Science Foundation of China (No. 30471510), and Liaoning Provincial Department of Education College Project Plan (No. L2012441, 2009S005). We would also like to thank our tutor Dr. Lun for the guidance of the relevant theories, experiment technology and experimental results of this experiment, as well as Dr. Sun Wuping, Professor Guo Ming, and other friends for their assistance with the experiment.

\section{References}

1. J. Wohlrab and D. Kreft, Skin Pharmacol Physiol. 27, 311 (2014).

2. S.J. Yang, J.M. Choi, L Kim, S.E. Park, E.J. Rhee, W.Y. Lee, K.W. Oh, S.W. Park, and C.Y. Park, J Nutr Biochem. 25, 66 (2014).

3. SS Zhou, D Li, WP Sun, M Guo, YZ Lun, YM Zhou, FC Xiao, LX Jing, SX Sun, LB Zhang, N Luo, FN Bian, W Zou, LB Dong, ZG Zhao, SF Li, XJ Gong, ZG Yu, CB Sun, CL Zheng, DJ Jiang, and ZN Li, World J Gastroenterol.,15, 5674 (2009).

4. SS Zhou, D Li, YM Zhou, WP Sun, XX Liu, and YZ Lun, Acta Physiologica Sinica. 62, 86 (2010).

5. K. Ortmayr, J. Nocon, B. Gasser, D. Mattanovich, S. Hann, and G. Koellensperger, J Sep Sci. 37, 2185 (1024).

6. R. Lang, A. Wahl, T. Skurk, E.F. Yagar, L. Schmiech, R. Eggers, H. Hauner, and T. Hofmann,
Anal Chem. 82, 1486 (2010).

7. M. Szafarz, Lomnicka M, Sternak M, Chlopicki S, and J. Szymura-Oleksiak, J Chromatogr B Analyt Technol Biomed Life Sci. 878, 895 (201011-12).

8. Jia Woei Wong, Kah Hay Yuen, and Kok Khiang Peh, J Chromatogr B Biomed Sci Appl. 716, 387 (1998).

9. A. Mancinelli, S. Pace, A. Marzo, E. A. Martelli , and G. Passetti, J Chromatogr. 575, 101 (1992).

10. V.M. Kodentsova, O.A. Vrzhesinskaia, A.A. Sokol'nikov, T.G. Zaburkina, E.D. Bender, and V.B. Spirichev, Vopr Pitan. 2, 62 (1992).

11. D. Zhong, and X. Chen, J Chromatogr B Biomed Sci Appl. 721, 67 (1999).

12. G.L. Chan, J.E. Axelson, F.S. Abbott, C.R. Kerr, and K.M. McErlane, J Chromatogr. 417, 295 (1987).

13. B. Alpertunga, S. Sungur, L. Ersoy, and S.Y .Manav, Arch Pharm (Weinheim). 323, 587 (1990).

14. T. Hirayama, K. Yoshida, K. Uda, M. Nohara, and S. Fukui. Anal Biochem. 147, 108 (1985).

15. B.R. Clark, Methods Enzymol. 66, 5(1980).

16. B.R. Clark, R.M. Halpem, and R.A. Smith, Anal Biochem. 68, 54 (1975).

17. C. Musfeld, J. Biollaz, N. Bélaz, U. W. Kesselring, and L.A. Decosterd, J Pharm Biomed Anal. 24, 391 (2001).

18. L.A. Decosterd, A. Karagiannis, J.M. Roulet, N. Bélaz, M. Appenzeller, T. Buclin, P. Vogel, and J. Biollaz, J Chromatogr B Biomed Sci Appl. 703, 25 (1997).

19. Yu H, Cai Y, Bi S, and Huang D, Se Pu. 23, 114 (2005).

20. Wang A, Li G, You J, Ji Z. J Sep Sci. 36, 3853 (2013). 